\title{
Treinamento muscular na face: a prática dos fonoaudiólogos de Belo Horizonte
}

\author{
Facial muscular training: the practice of speech-language pathologists \\ from Belo Horizonte
}

\author{
Grazielle Costa Coutrin ${ }^{1}$, Luciana Ulhôa Guedes², Andréa Rodrigues Motta ${ }^{3}$
}

\begin{abstract}
RESUMO
Objetivo: Verificar a prática do fonoaudiólogo que atua em motricidade orofacial em Belo Horizonte, acerca de treinamento muscular na face e comparar a conduta de profissionais com e sem especialização na área. Métodos: Estudo transversal, com aplicação de questionário a 60 fonoaudiólogos de Belo Horizonte, atuantes em motricidade orofacial, sendo 30 sem especialização e 30 com especialização na área. O instrumento apresentou a descrição de um caso clínico e 12 perguntas acerca de condutas em mioterapia. Para análise dos dados foram empregados os testes qui-quadrado e exato de Fisher, sendo considerado um nível de significância de 5\%. Resultados: Na amostra pesquisada, 88,3\% citaram exercícios isométricos, 63,3\% indicaram freqüência de treinamento de três vezes ao dia e $81,7 \%$ sete dias por semana. A variação do tempo de contração muscular foi citada por $61,7 \%$, sendo que $40,0 \%$ orientam aumento do tempo. Indicaram necessidade de variar o número de séries dos exercícios ao longo da terapia 50,0\%, sendo que $26,7 \%$ destes citaram a diminuição. Quanto à frequiência, 43,3\% consideraram necessário realizar variação ao longo da terapia, tendo 20,0\% informado diminuir o número de vezes por dia e 23,3\% diminuir o número de dias por semana. O tempo médio de quatro a seis meses para fonoterapia foi citado por 56,7\% dos fonoaudiólogos. Conclusão: A maioria dos fonoaudiólogos citou empregar exercício isométrico, três vezes ao dia, sete dias por semana, com tempo médio de terapia entre quatro e seis meses. A conduta dos fonoaudiólogos com e sem especialização não apresentou diferença significante.
\end{abstract}

Descritores: Contração muscular; Força muscular; Terapia miofuncional; Prática profissional; Questionários

\section{INTRODUÇÃO}

Historicamente o treinamento físico é uma atividade muito antiga; entretanto, seu corpo de conhecimento é relativamente recente. Um desenvolvimento acelerado dos conhecimentos teóricos e práticos sobre o processo do treinamento ocorreu na metade do século XX, sendo que conceitos que tradicionalmente eram aplicados aos esportes, foram estendidos à saúde, à terceira idade, à reabilitação, entre outras áreas ${ }^{(1)}$.

O fonoaudiólogo utiliza duas formas de trabalho para mo-

Trabalho realizado na Universidade Federal de Minas Gerais - UFMG - Belo Horizonte (MG), Brasil.

(1) Fonoaudióloga clínica - Belo Horizonte (MG), Brasil.

(2) Mestre, Professora dos Cursos de Pós-graduação do Centro FEAD de Fonoaudiologia e Centro de Estudos Superiores de Juiz de Fora - Belo Horizonte (MG), Brasil.

(3) Pós-graduanda em Distúrbios da Comunicação Humana pela Universidade Federal de São Paulo - UNIFESP - São Paulo (SP), Brasil; Professora Assistente do Departamento de Fonoaudiologia da Universidade Federal de Minas Gerais - UFMG - Belo Horizonte (MG), Brasil.

Endereço para correspondência: Grazielle Costa Coutrin. R. Itapetininga, 1003, Bairro Cardoso, Belo Horizonte - MG, CEP 30626-470. E-mail: grazi_fono2006@yahoo.com.br

Recebido em: 3/10/2007; Aceito em 12/4/2008 dificações musculares: a mioterapia e a terapia miofuncional. Na mioterapia, ocorre a atuação específica no músculo que se quer modificar, utilizando-se exercícios isotônicos e/ou isométricos. Na terapia miofuncional, trabalha-se diretamente com as funções que se quer adequar atingindo com isto a modificação muscular. Apesar de a terapia miofuncional parecer mais rápida e eficiente, é reconhecida a importância de exercícios específicos em determinados momentos ${ }^{(2)}$.

A prática de exercícios é desenvolvida por meio de contrações de diversos grupos musculares. Os músculos esqueléticos apresentam dois tipos básicos de contração: a isotônica e a isométrica, conforme a mobilidade dos pontos de fixação deste músculo. Uma contração muscular, na qual uma extremidade do músculo está fixa e outra móvel, contra uma força constante é denominada isotônica. Por outro lado, quando as duas extremidades musculares estão fixas, impossibilitando a variação do comprimento muscular, temos uma contração denominada isométrica. A grande maioria das contrações não é puramente isométrica ou isotônica, mas sim um padrão misto das duas ${ }^{(3)}$.

A plasticidade muscular possibilita que a prática de exercícios tenha efeitos sobre a forma e a função dos músculos esqueléticos, mas para que isto ocorra é preciso compreender 
e respeitar os princípios do treinamento muscular ${ }^{(4)}$. De acordo com o American College of Sports Medicine, o treinamento físico corporal e a prática de exercícios consistem basicamente na aplicação de sobrecargas aos sistemas músculo-esquelético, cardiovascular e neuro-endócrino com o principal objetivo de ganho de força e conseqüentemente melhora da função dos músculos. Sobrecarga deve ser entendida como uma solicitação de função acima dos níveis de repouso, sendo, portanto, uma situação de estresse que leva à desestruturação tecidual, consumo de substratos energéticos, de enzimas e de outras substâncias essenciais, comprometendo a homeostase. Sabe-se que sobrecargas excessivas em intensidade ou volume podem levar a lesões ou disfunções, mas no caso do treinamento físico bem orientado, as sobrecargas são bem dosadas, progressivas e intermitentes ${ }^{(5)}$.

No treinamento, a força desenvolvida por um músculo é proporcional à quantidade de unidades motoras ativadas durante aquela contração muscular. Estas unidades motoras são recrutadas de acordo com o princípio do tamanho, isto é das menores para as maiores ${ }^{(5)}$. Os ganhos iniciais da musculatura que é submetida a um treinamento cujo objetivo seja ganhar força incluem adaptações neurais como aumento do recrutamento das fibras e da frequiência de descarga dos potenciais de ação, diminuição na co-contração da musculatura antagonista e aprendizagem do movimento. Apenas após 6-8 semanas de treino será observado aumento da área de secção transversa do músculo (hipertrofia) ${ }^{(1,5)}$. A magnitude destas mudanças depende dos princípios do regime de treinamento muscular, tais como: tipo de ação muscular, intensidade, volume, tipo de exercício, ordem dos exercícios, período de repouso entre as séries e frequiência ${ }^{(5)}$.

No intuito de manipular os estímulos de treinamento e alcançar melhores resultados, vários métodos de treinamento de força foram desenvolvidos. Os métodos manipulam as variáveis de treinamento de diferentes maneiras, fornecendo estímulos mecânicos e metabólicos de diferentes magnitudes. O estímulo mecânico é diretamente influenciado pela quantidade de peso levantada em cada repetição e pelo número de repetições feitas por série( ${ }^{(6)}$.

Os músculos faciais são músculos esqueléticos, porém possuem particularidades que os diferem e merecem especial atenção no planejamento terapêutico. Diferentemente dos demais músculos esqueléticos, não possuem fusos musculares. Possuem, ainda, unidades motoras pequenas, tendo uma relação de 25 fibras musculares por motoneurônio, o que permite maior complexidade de movimento. Entretanto, devido à proximidade e ao pequeno tamanho dos músculos faciais, se torna difícil a contração isolada. Sendo assim, o uso de técnicas de exercícios não específicos para a musculatura facial não seria eficaz no tratamento das alterações desses músculos, sendo de fundamental importância um tratamento com maior especificidade e adaptação às características únicas dos músculos da face ${ }^{(7)}$.

O fonoaudiólogo deve conhecer as potencialidades e as limitações orgânicas e funcionais capazes de ampliar ou limitar as possibilidades de tratamento. O sucesso da terapia em motricidade orofacial, por meio de treinamento muscular, depende da prescrição de exercícios e para que a mesma seja efetiva, é necessário respeitar os princípios de treinamento muscular. Porém, sabe-se que na literatura científica, estudos sobre o treinamento muscular na face são escassos. Até mesmo trabalhos que citem as metodologias empregadas na terapêutica mioterápica não são freqüentes ${ }^{(8-14)}$.

Desta forma, o objetivo do presente estudo foi verificar a prática dos fonoaudiólogos de Belo Horizonte, que atuam em motricidade orofacial, acerca do treinamento muscular na face e comparar a conduta de fonoaudiólogos com e sem especialização na área.

\section{MÉTODOS}

Este estudo transversal foi realizado com 60 fonoaudiólogos de Belo Horizonte, atuantes na área de motricidade orofacial (MO), sendo estes distribuídos em dois grupos: 30 graduados sem especialização em $\mathrm{MO}$ e 30 graduados com especialização em MO. Os fonoaudiólogos foram contatados em cursos na área e também selecionados por meio de sorteio a partir da lista telefônica.

Participaram do estudo os profissionais que obedeceram aos seguintes critérios de inclusão: a) fonoaudiólogos atuantes na área de motricidade orofacial; b) fonoaudiólogos atuantes na cidade de Belo Horizonte; c) fonoaudiólogos que autorizaram previamente a sua participação na pesquisa mediante assinatura do termo de consentimento livre e esclarecido.

Os critérios de exclusão estabelecidos na pesquisa foram: a) fonoaudiólogos atuantes na área de ensino superior em motricidade orofacial; b) fonoaudiólogos com graduação e/ou especialização em áreas de treinamento muscular (Fisioterapia e Educação Física).

A coleta de dados foi realizada por meio de questionário (Anexo 1). O local de aplicação do instrumento não foi padronizado, sendo escolhido individualmente pelos profissionais. O questionário apresentava a descrição de um caso clínico de hipotensão de orbicular da boca, não decorrente de alteração oclusal e 12 perguntas, quatro fechadas e oito abertas, referentes ao tipo de exercício a ser proposto àquele caso; o tempo de contração muscular, o número de séries, a frequiência dos exercícios, existência ou não de variação gradual nos exercícios ao longo da terapia e o tempo médio de terapia para pacientes de motricidade orofacial.

Cada profissional descreveu um exercício a ser empregado e, em seguida, especificou parâmetros de treinamento muscular para o mesmo. Para classificação dos exercícios em isométricos ou isotônicos, observou-se a descrição do exercício ${ }^{(15)} \mathrm{o}$ tempo de contração muscular citado.

A metodologia proposta para esse trabalho foi testada em um estudo piloto. A intenção inicial era avaliar a objetividade e clareza do questionário. Tendo em vista que os participantes não apresentaram nenhuma dificuldade na compreensão do caso clínico e/ou das perguntas, o questionário foi mantido.

As informações obtidas por meio do questionário foram contabilizadas, de forma a determinar dados quantitativos, assim como estabelecer possíveis relações entre fonoaudiólogos com e sem especialização em MO e as condutas indicadas no treinamento muscular da face. As variáveis discretas foram analisadas por medidas de tendência central, já as categóricas 
foram inicialmente estudadas por análise de freqüiência, tendo sido em seqüências agrupadas em respostas dicotômicas. Nesta segunda etapa, foram utilizados o teste exato de Fisher e o teste qui-quadrado, por meio do programa Epi-Info (6.0), sendo considerado um nível de significância de 5\% ( $<<0,05)$. Essa pesquisa foi aprovada pelo Comitê de Ética e Pesquisa da Universidade Federal de Minas Gerais, sob o parecer $n^{\circ}$ ETIC 375/05.

\section{RESULTADOS}

O gênero feminino foi observado em $100 \%$ da amostra, sendo que a idade das entrevistadas variou entre 22 e 53 anos, com média de 28,5 anos. A faixa etária de 20 a 30 anos foi a mais prevalente $(68,3 \%)$. Quanto ao ano de conclusão da graduação $60 \%$ dos fonoaudiólogos sem especialização em MO concluíram o curso de Fonoaudiologia no período de 2003 a 2005 enquanto $40 \%$ com especialização em MO no período de 2000 a 2002.

Os dados referentes ao tipo de exercício empregado para o caso clínico apresentado encontram-se na Tabela 1. Ao se associar as variáveis, presença de especialização e tipo de exercício, agrupando-se as respostas nas variáveis dicotômicas, isométrico e não isométrico, não se observou, pelo teste exato de Fisher, associação significante $(\mathrm{p}=0,664)$ entre ser ou não especialista em MO.

Em relação ao tempo de contração a ser indicado num primeiro momento para o caso clínico, verificou-se entre as profissionais sem especialização média de 10,4 segundos, mediana de 10 segundos e desvio padrão de 10,9, indicando uma grande heterogeneidade nos dados. Respostas extremas foram observadas, tendo variado os dados de 2 a 60 segundos. Entre os fonoaudiólogos com especialização, encontraram-se dados um pouco mais homogêneos com média de 7,7 segundos, mediana de 5 segundos e desvio padrão de 3,6. Na análise do número inicial de séries a ser prescrito para o caso, verificou-se dentre os profissionais sem especialização média de 1,2 séries $\pm 0,5$, com mediana de 1 série e entre os especialistas, 2 séries \pm 2 , com mediana de 1 série.

Em seguida, serão apresentados os dados sobre a opinião da amostra no que se refere à importância e forma de variação dos parâmetros durante o treinamento muscular. Na Tabela 2 podem ser observados os dados referentes à freqüência de treinamento em número de vezes do exercício indicado, por dia. Agrupando-se as respostas em três vezes ao dia ou outra opção, o teste qui-quadrado não indicou diferenças entre especialistas e não especialistas $(\mathrm{p}=0,592)$.

Todos os entrevistados citaram indicar a continuidade do treinamento em casa. A freqüência deste treinamento é apresentada na Tabela 3. Pelo teste qui-quadrado, as respostas sete dias por semana ou menos de sete dias, não apresentaram diferença significante entre os grupos pesquisados $(\mathrm{p}=1,000)$.

Quanto à necessidade de se variar o tempo de contração muscular ao longo da terapia, $14(46,7 \%)$ profissionais com

Tabela 1. Distribuição dos fonoaudiólogos quanto ao tipo de exercício empregado

\begin{tabular}{|c|c|c|c|c|c|c|c|}
\hline \multirow{3}{*}{$\begin{array}{l}\text { Tipo de } \\
\text { exercício } \\
\text { empregado }\end{array}$} & \multicolumn{6}{|c|}{ Fonoaudiólogos de Belo Horizonte } & \multirow{3}{*}{$p$-valor* } \\
\hline & \multicolumn{2}{|c|}{$\begin{array}{l}\text { Sem especialização em } \\
\text { Motricidade Orofacial }\end{array}$} & \multicolumn{2}{|c|}{$\begin{array}{l}\text { Com especialização em } \\
\text { Motricidade Orofacial }\end{array}$} & \multicolumn{2}{|c|}{ Total } & \\
\hline & $\mathrm{N}$ & $\%$ & $\mathrm{~N}$ & $\%$ & $\mathrm{~N}$ & $\%$ & \\
\hline Isotônico & 3 & 10,0 & 1 & 3,3 & 4 & 6,7 & - \\
\hline Isométrico & 27 & 90,0 & 27 & 90,0 & 54 & 90,0 & 0,664 \\
\hline Treino funcional & 0 & 0,0 & 2 & 6,7 & 2 & 3,3 & - \\
\hline Total & 30 & 100,0 & 30 & 100,0 & 60 & 100,0 & \\
\hline
\end{tabular}

Legenda: * Teste exato de Fisher

Tabela 2. Distribuição dos fonoaudiólogos quanto à freqüência de treinamento em número de vezes do exercício por dia

\begin{tabular}{|c|c|c|c|c|c|c|c|}
\hline \multirow{3}{*}{$\begin{array}{l}\text { Freqüência de } \\
\text { treinamento } \\
\left(n^{\circ} x / \text { dia }\right)\end{array}$} & \multicolumn{6}{|c|}{ Fonoaudiólogos de Belo Horizonte } & \multirow{3}{*}{$\mathrm{p}$-valor } \\
\hline & \multicolumn{2}{|c|}{$\begin{array}{l}\text { Sem especialização em } \\
\text { Motricidade Orofacial }\end{array}$} & \multicolumn{2}{|c|}{$\begin{array}{l}\text { Com especialização em } \\
\text { Motricidade Orofacial }\end{array}$} & \multicolumn{2}{|c|}{ Total } & \\
\hline & $\mathrm{N}$ & $\%$ & $\mathrm{~N}$ & $\%$ & $\mathrm{~N}$ & $\%$ & \\
\hline 1x/dia & 4 & 13,3 & 5 & 16,7 & 9 & 15,0 & - \\
\hline $2 x /$ dia & 4 & 13,3 & 4 & 13,3 & 8 & 13,3 & - \\
\hline $3 x /$ dia & 20 & 66,7 & 18 & 60,0 & 38 & 63,3 & 0,592 \\
\hline $4 \mathrm{x} / \mathrm{dia}$ & 1 & 3,3 & 2 & 6,7 & 3 & 5,0 & - \\
\hline $5 x /$ dia & 0 & 0,0 & 1 & 3,3 & 1 & 1,7 & - \\
\hline $\mathrm{NI}$ & 1 & 3,3 & 0 & 0,0 & 1 & 1,7 & - \\
\hline Total & 30 & 100,0 & 30 & 100,0 & 60 & 100,0 & \\
\hline
\end{tabular}

Legenda: * Teste qui-quadrado; $\mathrm{NI}=$ não informado; $\mathrm{x} / \mathrm{dia}=$ vezes ao dia 
Tabela 3. Distribuição dos fonoaudiólogos quanto à freqüência de treinamento em número de dias por semana

\begin{tabular}{|c|c|c|c|c|c|c|c|}
\hline \multirow{3}{*}{$\begin{array}{l}\text { Freqüência de } \\
\text { treinamento } \\
\left(n^{\circ} \mathrm{d} / \mathrm{sem}\right)\end{array}$} & \multicolumn{6}{|c|}{ Fonoaudiólogos de Belo Horizonte } & \multirow{3}{*}{ p-valor } \\
\hline & \multicolumn{2}{|c|}{$\begin{array}{l}\text { Sem especialização em } \\
\text { Motricidade Orofacial }\end{array}$} & \multicolumn{2}{|c|}{$\begin{array}{l}\text { Com especialização em } \\
\text { Motricidade Orofacial }\end{array}$} & \multicolumn{2}{|c|}{ Total } & \\
\hline & $\mathrm{N}$ & $\%$ & $\mathrm{~N}$ & $\%$ & $\mathrm{~N}$ & $\%$ & \\
\hline $7 d /$ sem & 24 & 80,0 & 25 & 83,3 & 49 & 81,7 & 1,000 \\
\hline $6 \mathrm{~d} / \mathrm{sem}$ & 1 & 3,3 & 2 & 6,7 & 3 & 5,0 & - \\
\hline $5 d / s e m$ & 5 & 16,7 & 1 & 3,3 & 6 & 10,0 & - \\
\hline $4 d /$ sem & 0 & 0,0 & 0 & 0,0 & 0 & 0,0 & - \\
\hline $3 d / s e m$ & 0 & 0,0 & 2 & 6,7 & 2 & 3,3 & - \\
\hline Total & 30 & 100,0 & 30 & 100,0 & 60 & 100,0 & \\
\hline
\end{tabular}

Legenda: * Teste qui-quadrado; $\mathrm{d} / \mathrm{sem}=$ dias por semana

especialização e $22(73,3 \%)$ sem especialização afirmaram ser importante esta variação, sendo a forma empregada para tanto apresentada na Figura 1. Considerando-se as variáveis dicotômicas manter ou variar o tempo de contração, mais uma vez não foram observadas diferenças significantes pelo teste qui-quadrado ( $\mathrm{p}=0,184)$.

No que diz respeito à necessidade de se variar o número de séries do exercício ao longo da terapia, metade $(50,0 \%)$ dos participantes de cada grupo afirmou ser tal fato importante. A forma empregada nesta variação está presente na Figura 2. Não foi verificada associação significante (qui-quadrado: $p=$ 0,796 ) entre o fato de manter ou variar as séries e o fato de ser ou não especialista em MO.

Quanto à necessidade de se variar a freqüência dos exercícios ao longo da terapia, $10(33,3 \%)$ especialistas e $16(53,3 \%)$ fonoaudiólogos sem especialização acreditam ser esta variação importante. A forma empregada para esta variação por número

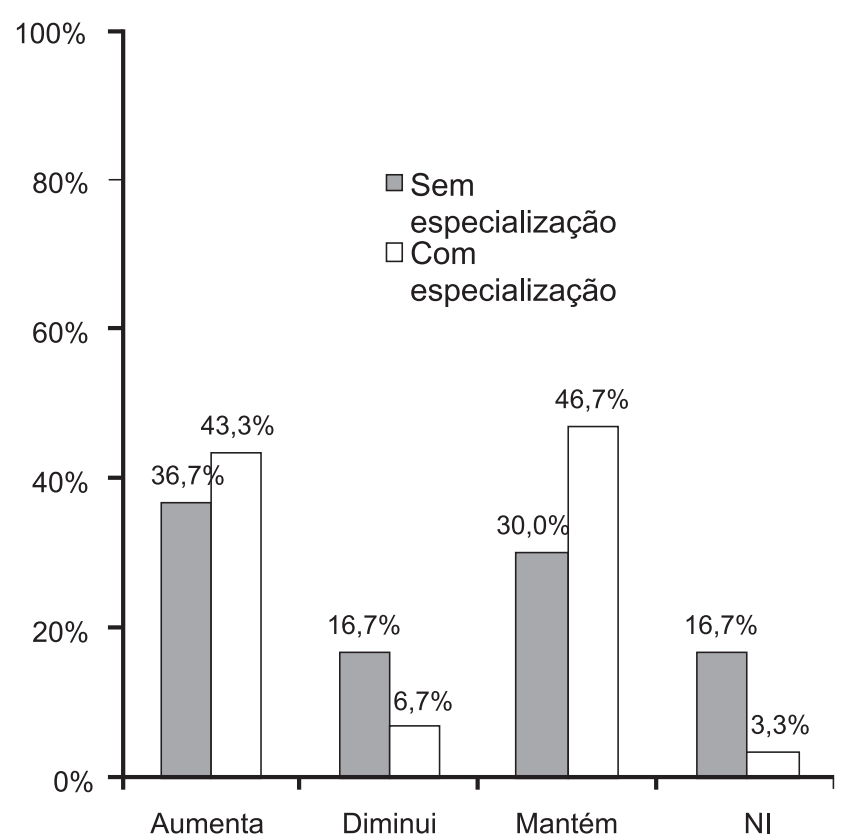

Figura 1. Distribuição dos fonoaudiólogos quanto à forma de variação do tempo de contração muscular ao longo da terapia.

Legenda: $\mathrm{NI}=$ não informado de vezes por dia está descrita na Figura 3 e por número de dias por semana na Figura 4. A associação das variáveis manter ou variar a frequiência de treinamento durante o dia $(\mathrm{p}=0,190)$ ou durante os dias da semana $(\mathrm{p}=0,436)$ não foi significante por meio do teste qui-quadrado.

Por fim, em relação ao tempo médio de terapia em MO citado pelos fonoaudiólogos pesquisados, os dados são apresentados na Tabela 4. A associação das variáveis agrupando-se os dados em 4-6 meses e outra opção, não indicou diferenças pelo teste qui-quadrado $(\mathrm{p}=0,190)$, entre especialistas e não especialistas em MO.

\section{DISCUSSÃO}

Os princípios de treinamento muscular são fundamentais para o sucesso de um trabalho, seja ele reabilitador ou não. No planejamento do treinamento corporal que busque melhorar a

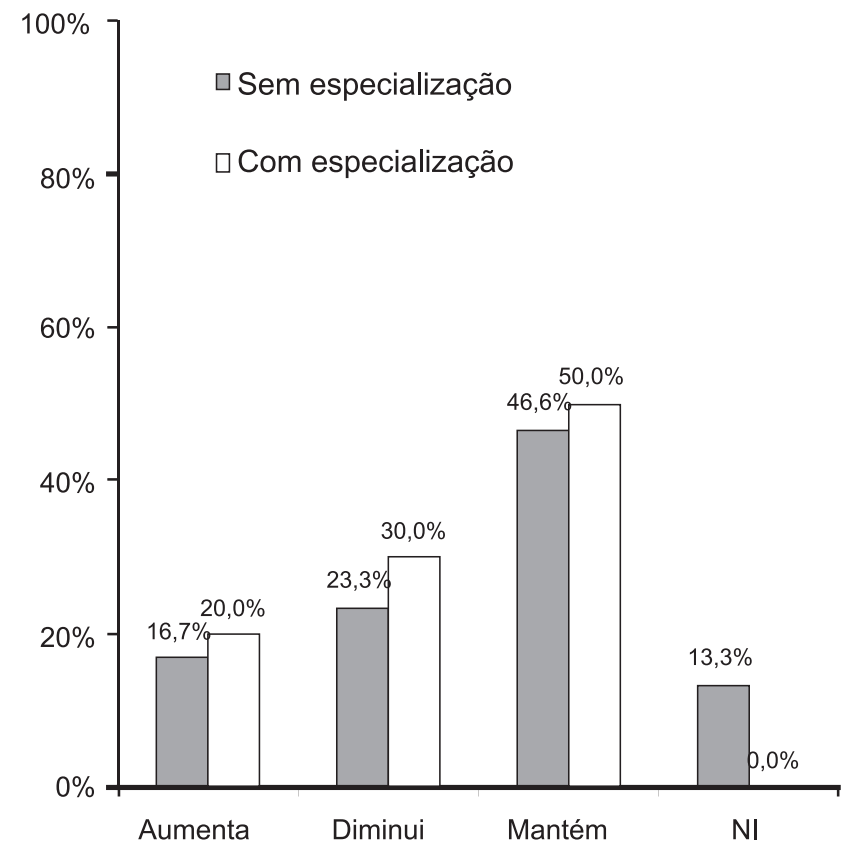

Figura 2. Distribuição dos fonoaudiólogos quanto à forma de variação do número de séries do exercício ao longo da terapia.

Legenda: $\mathrm{NI}=$ não informado 


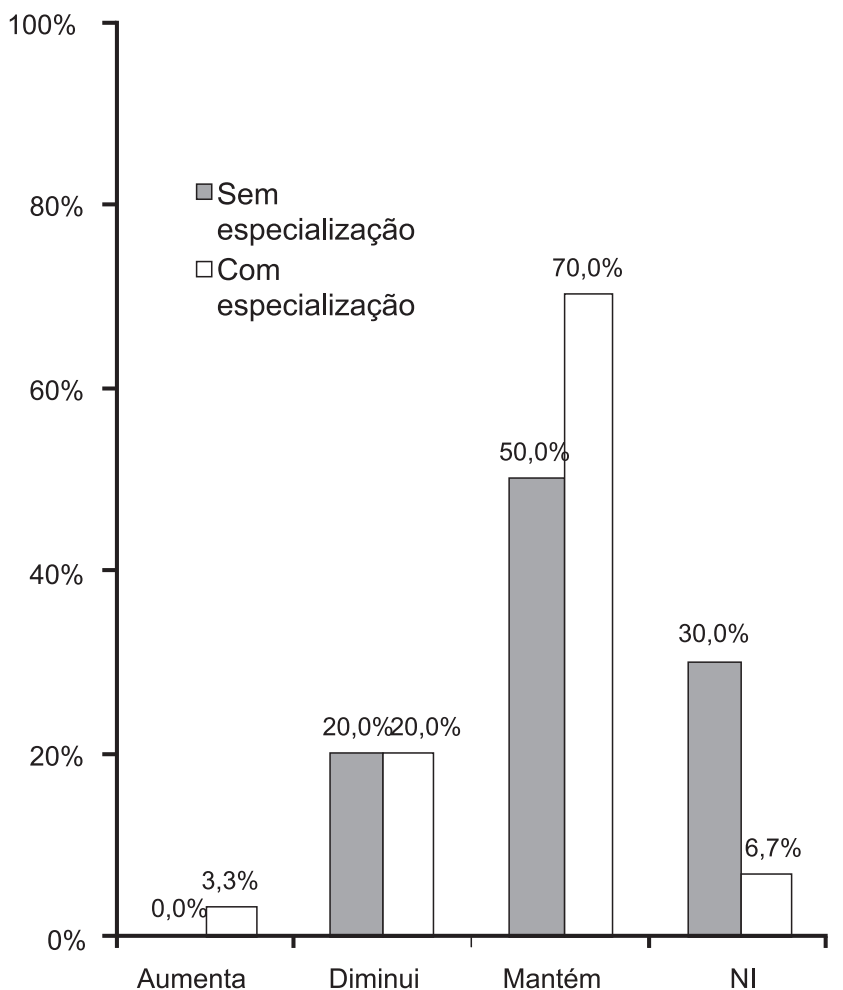

Figura 3. Distribuição dos fonoaudiólogos quanto à forma de variação da freqüência do exercício em número de vezes por dia ao longo da terapia.

Legenda: $\mathrm{NI}=$ não informado

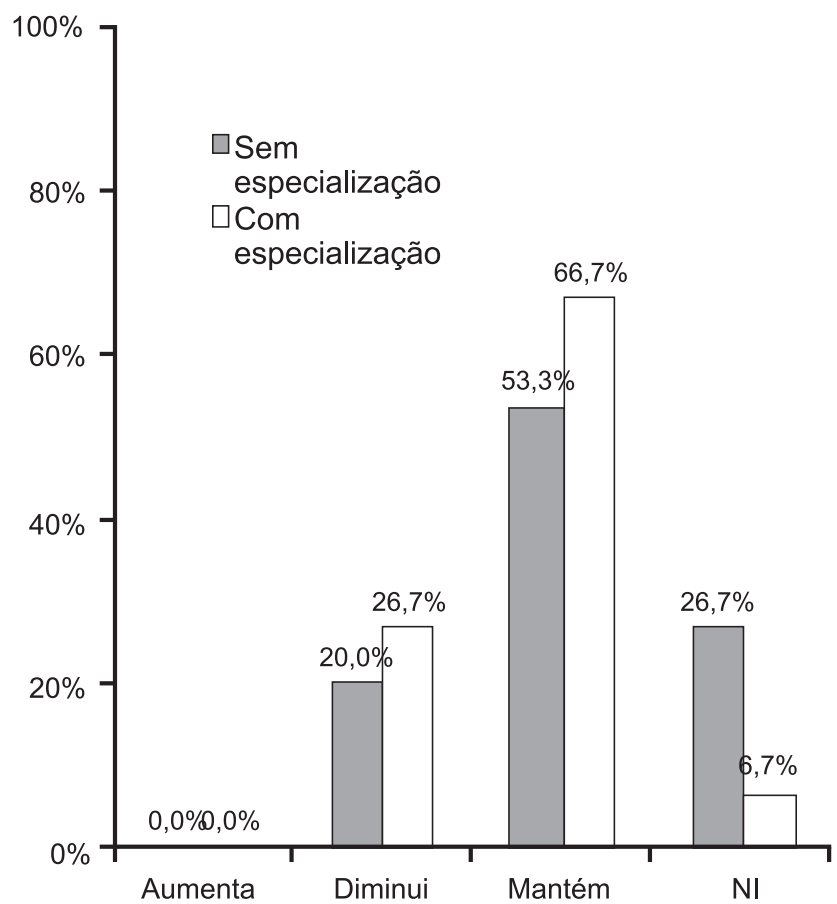

Figura 4. Distribuição dos fonoaudiólogos quanto à forma de variação da freqüência do exercício em número de dias por semana ao longo da terapia.

Legenda: $\mathrm{NI}=$ não informado

Tabela 4. Distribuição dos fonoaudiólogos quanto ao tempo médio de terapia fonoaudiológica nos distúrbios miofuncionais orofaciais e cervicais

\begin{tabular}{|c|c|c|c|c|c|c|c|}
\hline \multirow{3}{*}{$\begin{array}{l}\text { Tempo médio } \\
\text { de terapia }\end{array}$} & \multicolumn{6}{|c|}{ Fonoaudiólogos de Belo Horizonte } & \multirow{3}{*}{$\mathrm{p}$-valor* } \\
\hline & \multicolumn{2}{|c|}{$\begin{array}{l}\text { Sem especialização em } \\
\text { Motricidade Orofacial }\end{array}$} & \multicolumn{2}{|c|}{$\begin{array}{l}\text { Com especialização em } \\
\text { Motricidade Orofacial }\end{array}$} & \multicolumn{2}{|c|}{ Total } & \\
\hline & $\mathrm{N}$ & $\%$ & $\mathrm{~N}$ & $\%$ & $\mathrm{~N}$ & $\%$ & \\
\hline $1-3$ meses & 13 & 43,3 & 11 & 36,7 & 24 & 40,0 & - \\
\hline 4-6 meses & 15 & 46,7 & 19 & 63,3 & 34 & 56,7 & 0,190 \\
\hline 7-9 meses & 1 & 3,3 & 0 & 0,0 & 1 & 1,7 & - \\
\hline 12 meses & 1 & 3,3 & 0 & 0,0 & 1 & 1,7 & - \\
\hline Total & 30 & 100,0 & 30 & 100,0 & 60 & 100,0 & \\
\hline
\end{tabular}

Legenda: * Teste qui-quadrado

força, deve-se levar em conta o tipo de ação muscular, intensidade, volume, tipo de exercício, ordem dos exercícios, período de recuperação entre as séries, periodicidade, número de séries e freqüência ${ }^{(1,5)}$. Observa-se que quando tais princípios são levados em consideração, os resultados são mais satisfatórios e o trabalho muscular ocorre de forma mais eficaz.

Ao se analisar os dados referentes ao conhecimento dos fonoaudiólogos acerca dos parâmetros de treinamento muscular, assim como da necessidade e da forma de variação dos mesmos, verificou-se pouca aplicação desses parâmetros na prática clínica. Curiosamente, os profissionais sem especialização em MO indicaram realizar mais variações nos treinamentos mioterápicos, entretanto não se observou diferença estatística. O fato de $60 \%$ destes fonoaudiólogos haverem concluído o cur- so de graduação entre os anos de 2003 e 2005, pode justificar tal tendência, já que nos últimos anos houve um avanço nas pesquisas cientificas na área e os cursos de graduação cada vez mais tem facilitado o acesso a esse tipo de informação. Assim, ressalta-se a importância dos fonoaudiólogos buscarem por meio da Educação Continuada um enriquecimento profissional sendo capaz de acompanhar os avanços da ciência depois de concluída a formação básica.

Apesar da grande maioria dos fonoaudiólogos com e sem especialização em MO terem proposto exercícios de contração muscular isométrica para o caso clínico apresentado, foram citados exercícios de contração muscular isotônica. Alguns estudos $^{(11,13-14,16)}$ empregaram em sua metodologia somente exercícios de contração isométrica para o tratamento das 
alterações de hipotonia/hipofunção para casos de paralisia facial periférica, questões estéticas e terapia miofuncional em crianças e obtiveram resultados satisfatórios. Sabe-se que os exercícios de contração isotônica são utilizados principalmente para promover aumento da mobilidade ${ }^{(5)}$, sendo inclusive empregados na fase de seqüelas nas paralisias faciais periféricas, promovendo melhor mobilidade dos órgãos fonoarticulatórios e diminuindo as chances de posteriores sincinesias ${ }^{(17-18)}$.

No treinamento muscular, os conceitos de recrutamento de fibras musculares e sobrecargas progressivas, auxiliam na promoção da adequação da força muscular, sendo indicada na literatura, a utilização de exercícios isotônicos e isométricos de forma hierárquica. Assim, os isotônicos por exigirem um tempo de contração muscular menor (sendo, portanto, mais fáceis), devem ser realizados primeiro com objetivo de preparar a musculatura para um posterior tempo de contração mais prolongado, quando são inseridos os exercícios isométricos ${ }^{(5)}$. Entretanto, alguns estudos em Fonoaudiologia indicam a utilização dos exercícios isotônicos e isométricos associados, sem considerar tal hierarquia, orientando a prática dos dois tipos de exercícios simultaneamente ${ }^{(8-10,12)}$.

É importante salientar que apesar da maioria dos profissionais terem citado a utilização de exercícios isométricos, alguns dos exercícios propostos para o caso clínico recrutam um número reduzido de fibras musculares ${ }^{(15)}$. Este fato pode comprometer a eficácia do exercício no treino daquele grupo muscular específico, uma vez que a força desenvolvida por um músculo é proporcional à quantidade de unidades motoras ativadas durante a contração muscular ${ }^{(5)}$. Verificou-se também emprego de treino funcional por parte de especialistas, embora o caso citado tenha se referido a um adulto e a literatura indicar que apesar de a terapia miofuncional parecer mais rápida e eficiente, é reconhecida a importância de exercícios específicos em determinados momentos ${ }^{(2)}$.

Dentre os profissionais sem especialização, apenas quatro $(13,3 \%)$ citaram exercícios de contra resistência, sendo que esse número se eleva para $17(56,7 \%)$ dentre os especialistas. Acredita-se que exercícios de contra-resistência sejam importantes no trabalho de fortalecimento muscular; sabe-se que os exercícios no treino corporal são executados com auxílio de sobrecargas progressivas empregadas em contra resistência ao movimento ${ }^{(5)}$. Entretanto, a Fonoaudiologia não possui ainda qualquer procedimento para determinar a carga adequada ao treinamento muscular ou mesmo controlar sua variação, fatores fundamentais a serem considerados em relação à sobrecarga. De acordo com os conceitos de treinamento muscular sobrecargas excessivas em intensidade ou volume podem levar a lesões ou disfunções musculares ${ }^{(5,19)}$.

Os dados referentes ao tempo de contração muscular indicado para o caso apresentado demonstraram uma variação maior nas respostas dos profissionais sem especialização, sem diferença entre os grupos. Apesar da ausência de dados na literatura, os especialistas utilizam um critério mais uniforme para selecionar o tempo inicial, em um tratamento para fortalecimento do orbicular da boca. Já o número de séries prescritas neste momento inicial não variou tanto, sendo uma tendência geral iniciar com um número pequeno. Esta conduta provavelmente levou em consideração que as sobrecargas aplicadas devem ser progressivas ${ }^{(5)}$.

Dentre os entrevistados, grande parte relatou solicitar uma freqüência de treinamento de três vezes ao dia. Concordando com a maioria dos profissionais do presente estudo, uma pesquisa cujo objetivo foi avaliar os efeitos da utilização de um dispositivo de resistência muscular, Face Former Therapy ${ }^{\circledR}$, na terapia miofuncional em crianças, obteve respostas satisfatórias com uma frequiência de treinamento dos exercícios de três vezes por $\mathrm{dia}^{(14)}$. Entretanto, outros estudos empregaram uma frequiência de duas vezes por dia, com melhoras significativas no restabelecimento miofuncional ${ }^{(13,16,20-21)}$. Alguns estudos, porém, utilizaram em sua metodologia a padronização do número de séries de cada exercício que poderiam ou não ser divididas durante o dia ${ }^{(8,10,12)}$. Os fundamentos de treinamento muscular nos grandes grupos musculares empregam cargas elevadas e indicam a necessidade de um período de repouso ${ }^{(4-5)}$. Quando um treinamento excessivo e prolongado é aplicado simultaneamente a uma inadequada recuperação, muitas das alterações fisiológicas positivas associadas ao treinamento físico são revertidas ${ }^{(19)}$.

Observou-se que $100 \%$ dos profissionais orientam a realização dos exercícios em casa. Quanto à freqüência de treinamento em número de dias por semana, a maioria dos fonoaudiólogos de ambos os grupos orienta a realização dos exercícios sete dias por semana. Em várias pesquisas com objetivo de favorecer um restabelecimento miofuncional orofacial, a metodologia quanto à frequiência dos exercícios em número de dias por semana, concorda com a maioria dos profissionais do presente estudo ${ }^{(8,10-12,14,16,20-21)}$.

Quando a intensidade, a duração e a carga de trabalho diário dos exercícios são apropriadas, adaptações fisiológicas positivas ocorrem. Entretanto, existe uma linha muito tênue entre um ótimo desempenho e uma diminuição do mesmo em função do sobretreinamento, que pode incluir lesão e fraqueza muscular. Até o presente momento não existe um simples marcador que possa prever o sobretreinamento; no entanto, um controle adequado do desempenho físico ainda é considerado o padrão-ouro na detecção do mesmo ${ }^{(19)}$.

No caso da musculatura esquelética corporal, quando o objetivo é ganho de força e conseqüente melhora da função, a prática de exercícios é recomendada com uma frequiência de dois a três dias por semana para aqueles indivíduos que iniciam o treinamento e de quatro a cinco dias por semana para níveis mais avançados de treinamento ${ }^{(5)}$. Essa prática ocorre respeitando o princípio de plasticidade tecidual, sendo que após um regime de exercícios, um período de 48 horas de repouso muscular deve acontecer para recuperação e reestruturação tecidual por meio de adaptações morfológicas e funcionais específicas ${ }^{(4)}$.

É importante salientar que os músculos faciais são músculos esqueléticos, porém, possuem particularidades tais como: ausência de fusos musculares, unidades motoras pequenas, maior proximidade e pequeno tamanho dos músculos tornando mais difícil a contração isolada ${ }^{(7)}$. Portanto, no planejamento de um programa de treinamento muscular para os músculos faciais tais particularidades devem ser observadas. Sabe-se que a literatura sobre treinamento muscular específico para este grupo muscular ainda é muito escassa. Sendo assim, 
cabe o questionamento da necessidade do uso de sobrecargas progressivas, com auxílio de contra-resistência para agilizar e aumentar a eficiência do treinamento muscular na face, além da utilização de um período de descanso entre os exercícios miofuncionais orofaciais para recuperação e reestruturação tecidual.

Vale ressaltar que um dos fatores que contribui para as dificuldades em se estabelecer um programa de treinamento muscular para os músculos faciais é a escassez de instrumentos para avaliação. A ausência de instrumentação para verificação da força dos músculos faciais torna impossível analisar a real efetividade das intervenções miofuncionais realizadas. $\mathrm{O}$ instrumento mais comumente citado na literatura para estudos da musculatura facial é a eletromiografia de superfície. O sinal gerado na eletromiografia constitui a soma dos potenciais de ação da unidade motora, captado por um eletrodo ${ }^{(22)}$. O uso da eletromiografia de superfície em pesquisas é recente e seu emprego na prática clínica é ainda restrito ${ }^{(23)}$. Estudos em grandes grupos musculares ${ }^{(22,24)}$ e nos levantadores da mandíbula ${ }^{(25)}$ têm sido conduzidos para verificar qual componente eletromiográfico poderia ser empregado como melhor elemento para predizer a força muscular. Entretanto, os procedimentos para avaliação dos músculos faciais e da cavidade oral por meio da eletromiografia ainda são complexos, uma vez que existem dificuldades de posicionamento dos eletrodos em músculos de pequenas dimensões e grande proximidade ${ }^{(26)}$.

Apesar da vasta literatura sobre a importância da variação dos parâmetros de treinamento muscular corporal ao longo do tempo a prática clínica dos fonoaudiólogos de Belo Horizonte indicou que muitos profissionais não levam em consideração estes aspectos. Apenas 37 (61,7\%) profissionais indicaram acreditar ser necessário variar o tempo de contração muscular. De forma contraditória, considerando-se apenas a análise de freqüência, os especialistas mostram menor conhecimento sobre as bases fisiológicas dos exercícios musculares. Em relação à forma de variar o tempo de contração muscular verificou-se um predomínio de respostas indicando a manutenção ou o aumento do tempo de contração.

No presente estudo, não se observou diferença quanto à necessidade de variar ou não o número de séries dos exercícios ao longo da terapia, ou seja, metade dos fonoaudiólogos não considera importante a variação de séries. Em relação à forma de realizar essa variação, naqueles que não mantém constante o número de séries, não se observou grandes diferenças entre aumentar ou diminuir o parâmetro. Apenas não especialistas $(13,3 \%)$ deixaram de responder esta questão.

Quanto à necessidade de variação da frequiência dos exercícios de uma forma geral (número de vezes do exercício por dia e número de dias por semana), $34(56,7 \%)$ profissionais citaram não ser necessário variar a freqüência dos exercícios ao longo da terapia. Este dado indica novamente que a maioria dos fonoaudiólogos não emprega, em sua prática, conceitos do treinamento muscular. Mais uma vez, um número maior de não especialistas indicaram a necessidade de se realizar a variação destes parâmetros, sem diferença estatística.

Em relação à forma de variar a freqüência dos exercícios em número de vezes do exercício por dia, mais profissionais com especialização indicaram manter constante o parâmetro.
Nove $(30 \%)$ profissionais sem especialização e dois $(6,7 \%)$ especialistas não responderam esta questão. Já em relação à forma de variar a freqüência dos exercícios em número de dias por semana, os fonoaudiólogos que não orientaram a manutenção citaram a diminuição do número de dias. Nesta questão, oito $(26,7 \%)$ fonoaudiólogos sem especialização e dois $(6,7 \%)$ com deixaram de responder. Estes dados de diminuição de freqüência de treinamento podem ser associados à melhora clínica do paciente e preparo para alta. Cabe ressaltar que vários profissionais, em especial não especialistas, não responderam as questões sobre como realizar as variações.

Observa-se que na literatura sobre o treinamento muscular corporal, a frequiência do treinamento é definida como o número de práticas do regime de exercícios em uma semana ${ }^{(5)}$ e não há orientação para que um mesmo exercício seja realizado várias vezes ao dia. Já na prática terapêutica em Fonoaudiologia para os músculos faciais, observamos tanto na literatura ${ }^{(14,16,20)}$ quanto no presente estudo, a orientação de exercícios varias vezes durante o dia. Como foi ressaltado anteriormente, a literatura específica ainda é muito escassa e seriam necessários mais estudos na área para padronização de um programa de treinamento específico para os músculos faciais.

No presente estudo observou-se que alguns profissionais citaram ser necessária uma variação dos parâmetros de treinamento muscular, porém estes não realizam essa variação na prática clínica mantendo o mesmo tempo de contração muscular, número de séries e frequiência dos exercícios ao longo da terapia. O tecido muscular esquelético tem a capacidade de se reestruturar após uma situação de estresse causada pelo exercício físico. Em tais situações, o tecido muscular responde ao estímulo com aumento de força e volume até atingir um estado de equilíbrio em que o estímulo não seja mais um fator estressante àquele grupo muscular e deixe de promover adaptações teciduais $^{(4)}$.

Sendo assim, pode-se inferir que um programa de terapia muscular na face que utilize sempre os mesmos exercícios sem variar os parâmetros de treinamento, pode não ser tão eficaz quanto um programa que utilize a variação desses parâmetros e que produza maior estresse no sistema neuro-muscular devido à rápida e contínua alteração dos estímulos. Acredita-se que os dados muitas vezes controversos, nas diferentes pesquisas realizadas na área de treinamento miofuncional orofacial e as insuficientes literatura e pesquisa cientifica especializada, bem como a falta de uma normalização e controle dos exercícios de motricidade orofacial, sejam responsáveis pela inúmera diversidade de atuação metodológica nos profissionais da área que até hoje se encontra centrada em uma metodologia terapêutica baseada na prática clínica.

Com relação ao tempo de terapia observou-se que, no total, $24(40,0 \%)$ profissionais relataram entre um e três meses e $34(56,7 \%)$ tempo médio de quatro a seis meses para terapia fonoaudiológica em motricidade orofacial, sendo que mais profissionais com especialização responderam entre 4-6 meses. O período de oito semanas de treinamento corporal com pesos parece ser suficiente para provocar ganhos significantes de força muscular, tanto em homens quanto em mulheres. Entretanto, as mulheres parecem ter um incremento relativo, superior aos homens, indicando maior potencial para desenvol- 
vimento da força muscular após curtos períodos de treinamento com pesos ${ }^{(27)}$. Em alguns estudos faciais compilados o tempo de tratamento realizado variou entre um e três meses ${ }^{(10-14)}$. Sabe-se, no entanto, que nesses estudos, diferentemente do que se espera na prática clínica, objetiva-se a experimentação e comprovação da eficácia de um método terapêutico, não sendo necessário o restabelecimento morfofuncional total do indivíduo, já que isso poderia demandar um tempo de terapia mais prolongado. Deve-se ressaltar que, um tempo de terapia prolongado, contribui para a desmotivação do paciente. Sendo assim, a expansão do conhecimento científico especializado possibilitará maior eficácia e objetividade do tratamento, reduzindo consequentemente o tempo de terapia.

Os dados encontrados no presente estudo só podem ser considerados como indicadores para novas pesquisas, uma vez que contou com reduzida amostra de uma única cidade. Com base na escassez de literatura especifica, nas controvérsias metodológicas encontradas nas pesquisas e nos dados obtidos por meio deste estudo, observa-se uma necessidade de ampliar o conhecimento cientifico em treinamento muscular na face. Para tanto, seria fundamental a realização de outros estudos, considerando-se a população de todo o país. Importante também seria a condução de ensaios clínicos para que a aplicação de cada parâmetro de treinamento muscular pudesse ser verificado na face, uma vez que pelas especificidades da musculatura facial não seria plausível a aplicação dos princípios corporais diretamente na face. Apenas desta forma será possível sistematizar a melhor forma de atuação terapêutica, criando assim na classe fonoaudiológica o saber necessário para o reconhecimento e crescimento da profissão. Entretanto, para tanto, instrumentos de avaliação específicos para avaliação específica da força muscular na face precisarão ser desenvolvidos.

\section{CONCLUSÕES}

A maioria dos fonoaudiólogos citou empregar exercício isométrico para terapia fonoaudiológica nos casos de hipotensão dos músculos faciais. No treinamento muscular, é orientada a realização dos exercícios com uma frequiência de três vezes ao dia, sete dias por semana e com tempo médio de terapia entre quatro e seis meses.

A conduta dos fonoaudiólogos de Belo Horizonte com e sem especialização em Motricidade orofacial diferiu em relação à necessidade de variar o tempo de contração muscular, a frequiência dos exercícios ao longo do dia e da semana; entretanto, estes dados não foram significantes.

\begin{abstract}
Purpose: To verify the practice of the speech-language pathologist who acts in orofacial myology in Belo Horizonte - MG (Brazil) concerning facial muscular training, and to compare orofacial myology specialists and nonspecialists' conducts. Methods: Sectional study, with application of a questionnaire to 60 speech-language pathologists (SLP) from Belo Horizonte - MG (Brazil) who attend orofacial myology patients: 30 graduate without specialization and 30 specialized in orofacial myology. The questionnaire presented a case report and 12 questions regarding conducts in myotherapy. Data analysis used Chi-square and Fisher's Exact Test, and considered a significance level of 5\%. Results: In the researched sample, $88.3 \%$ of the SLP mentioned isometric exercises, $63.3 \%$ requested a training frequency of three times a day and $81.7 \%$ seven days a week. The variation of time of muscular contraction was mentioned by $61.7 \%$, and $40 \%$ recommend time increase. They indicated the need to vary the number of series of exercises along the therapeutic process in $50 \%$ of the interviews, and $26.7 \%$ of these mentioned decrease of this number. Regarding frequency, $43.3 \%$ considered necessary to vary the frequency of exercises along the therapeutic process: $20 \%$ reducing the number of repetitions of the exercise per day and $23.3 \%$ reducing the number of days a week. More than half of the professionals (56.7\%) informed that the average time of intervention for orofacial myology varied from four to six months. Conclusion: The majority of the SLP interviewed for this study employ isometric exercises, three times a day, seven days a week, for an average period of four to six months of therapy. There was no significant difference between the conduct of specialized and nonspecialized speech-language pathologists.
\end{abstract}

Keywords: Muscle contraction; Muscle strength; Myofunctional therapy; Professional practice; Questionnaires

\title{
REFERÊNCIAS
}

1. Barbanti VJ, Tricoli V, Ugrinowitsch C. Relevância do conhecimento científico na prática do treinamento físico. Rev Paul Educ Fis. 2004;18(N Esp):101-9.

2. Marchesan IQ. Distúrbios da Motricidade orofacial. In: Russo ICP. Intervenção fonoaudiológica na terceira idade. Rio de Janeiro: Revinter; 199p. p.83-100.

3. Weisbrodt NW. Músculo estriado esquelético. In: Johnson LR, editor. Fundamentos de fisiologia médica. 2a ed. Rio de Janeiro: GuanabaraKoogan; 2000. p.95-103.
4. Silverthorn DU, coordenador. Fisiologia humana: uma abordagem integrada. 2a. ed. Barueri, SP: Manole; 2003. p.345-82.

5. Kraemer WJ, Adams K, Cafarelli E, Dudley GA, Dooly C, Feigenbaum MS, Fleck SJ, Franklin B, Fry AC, Hoffman JR, Newton RU, Potteiger J, Stone MH, Ratamess NA, Triplett-McBride T; American College of Sports Medicine. American College of Sports Medicine position stand. Progression models in resistance training for healthy adults. Med Sci Sports Exerc. 2002;34(2):364-80. 
6. Gentil P, Oliveira E, Fontana K, Molina G, Oliveira RJ, Bottaro M. Efeitos agudos de vários métodos de treinamento de força no lactato sanguíneo e características de cargas em homens treinados recreacionalmente. Rev Bras Med Esporte. 2006;12(6):303-7.

7. Diels HJ. New concepts in nonsurgical facial nerve rehabilitation. Adv Otolaryngol Head Neck Surg. 1995;9:289-315.

8. Jardini RSR. Uso do exercitador labial: estudo preliminar para alongar e tonificar os músculos orbiculares orais. Pro-Fono. 1999;11(1):8-12.

9. Meyer PG. Tongue lip and jaw differentiation and its relationship to orofacial myofunctional treatment. Int J Orofacial Myology. 2000;26:44-52.

10. Jardini RSR. Uso do exercitador facial: um estudo preliminar para fortalecer os músculos faciais. Pro-Fono. 2001;13(1):83-9.

11. Takacs AP, Valdrighi V, Assencio-Ferreira VJ. Fonoaudiologia e estética: unidas a favor da beleza facial. Rev CEFAC. 2002;4(2):111-6.

12. Jardini RSR. Avaliação eletromiográfica do músculo bucinador flácido usando o exercitador facial. Pro-Fono. 2002;14(3):331-42.

13. van Lieshout PH, Bose A, Namasivayam AK. Physiological effects of an 8-week mechanically aided resistance facial exercise program. Int J Orofacial Myology. 2002;28:49-73.

14. Korbmacher HM, Schwan M, Berndsen S, Bull J, Kahl-Nieke B. Evaluation of a new concept of myofunctional therapy in children. Int J Orofacial Myology. 2004;30:39-52.

15. Coutrin GC. Treinamento muscular na face: a prática dos fonoaudiólogos de Belo Horizonte [monografia]. Belo Horizonte: Universidade Federal de Minas Gerais; 2006.

16. Gomez MVSG, Bogar P, Bento RF, Miniti A. Exercícios miofaciais e paralisia facial idiopática: relato preliminar. Rev Bras Otorrinolaringol. 1996;62(4):322-30.

17. Ribeiro EC, Cassol M. Enfoque fisioterápico e fonoaudiológico na paralisia facial periférica. Arq Inter Otorrinolaringol. 1999;3(3):101-5.
18. Gomez MVSG, Vasconcelos LGE, Moraes MFBB. Trabalho miofuncional na paralisia facial periférica. Arq Int Otorrinolaringol. 1999;3(1):30-4.

19. Cunha GS, Ribeiro JL, Oliveira AR. Sobretreinamento: teorias, diagnóstico e marcadores. Rev Bras Med Esporte. 2006;12(5):297-302.

20. Altmann EBC, Ramos APF, Vaz ACN. Paralisia facial congênita: resultados com fonoterapia oromiofuncional. Fono Atual. 1999;3(8):14-8.

21. Bernardes DFF, Gomez MVSG, Pirana S, Bento RF. Functional profile in patients with facial paralysis treated in a myofunctional approach. Pro-Fono. 2004;16(2):151-8.

22. Staudenmann D, Kingma I, Daffertshofer A, Stegeman DF, van Dieën JH. Improving EMG-based muscle force estimation by using a highdensity EMG grid and principal component analysis. IEEE Trans Biomed Eng. 2006;53(4):712-9.

23. Gomes CF, Trezza EMC, Murade ECM, Padovani CR. Surface electromyography of facial muscles during natural and artificial feeding of infants. J Pediatr (Rio J). 2006;82(2):103-9.

24. Staudenmann D, Daffertshofer A, Kingma I, Stegeman DF, van Dieën JH. Independent component analysis of high-density electromyography in muscle force estimation. IEEE Trans Biomed Eng. 2007;54(4):751-4.

25. Ferrario VF, Sforza C, Zanotti G, Tartaglia GM. Maximal bite forces in healthy young adults as predicted by surface electromyography. J Dent. 2004;32(6):451-7.

26. Takada K, Yashiro K, Sorihashi Y, Morimoto T, Sakuda M. Tongue, jaw, and lip muscle activity and jaw movement during experimental chewing efforts in man. J Dent Res. 1996;75(8):1598-606.

27. Dias RMR, Cyrino ES, Salvador EP, Nakamura FY, Pina FLC, Oliveira AR. Impacto de oito semanas de treinamento com pesos sobre a força muscular de homens e mulheres. Rev Bras Med Esporte. 2005;11(4):224-8

Anexo 1. Questionário

Gênero: $\square \mathrm{M} \quad \square \mathrm{F} \quad$ Idade:__ anos

Ano de conclusão da graduação:

Ano de conclusão da Especialização em Motricidade Oral:

Você recebeu, em seu consultório, um paciente adulto, de 23 anos, sem alteração oclusal, que apresenta hipotensão (hipotonia) moderada dos músculos orbiculares superior e inferior da boca.

Descreva um exercício que você indicaria para o caso:

A) Qual o tempo de contração muscular seria orientado inicialmente?

B) Que o número de séries seria orientado inicialmente?

1. Qual freqüência de treinamento muscular você indica? ( $n^{\circ}$ de vezes por dia)

2. Você orienta seus pacientes a fazerem os exercícios em casa? $\square$ Não $\square$ Sim. Com qual a freqüência

( $\mathrm{n}^{\circ}$ de dias por semana)?

3. Você acha necessário variar o tempo de contração muscular ao longo da terapia? $\square$ Não $\square$ Sim

4. Para o caso descrito, como você faria essa variação?

5. Você acha necessário variar o número de séries do exercício ao longo da terapia? $\square$ Não $\square$ Sim

6. Para o caso descrito, como você faria essa variação?

7. Você acha necessário variar a freqüência dos exercícios ao longo da terapia? $\square$ Não $\square$ Sim

8. Para o caso descrito, como você faria essa variação?

9. Considerando-se as inúmeras variáveis presentes no tratamento fonoaudiológico, qual o tempo de terapia em média para um paciente com distúrbio miofuncional orofacial e cervical? 